\title{
Manejo Quirúrgico de la Hiperplasia Condilar Tipo 2: Reporte de un Caso
}

\author{
Surgical Management of Mandibular Condylar Hyperplasia Type 2: Case Report
}

Víctor Hiram Barajas-Pérez'; Marcelo Enrique Cazar-Almache²; José Ernesto Miranda-Villasana; Norith de Jesús Recendez-Santillan; Juan Carlos Cuevas-González ${ }^{5}$ \& Graciela Zambrano Galván ${ }^{6}$

\begin{abstract}
BARAJAS-PÉREZ, V. H.; CAZAR-ALMACHE, M. E.; MIRANDA-VILLASANA, J. E.; RECENDEZ-SANTILLAN, N. J.; CUEVAS-GONZÁLEZ, J. C. \& ZAMBRANO, G. G. Manejo quirúrgico de la hiperplasia condilar tipo 2: Reporte de un caso. Int. J. Odontostomat., 13(1):5-10, 2019.

RESUMEN: La hiperplasia condilar $(\mathrm{HC})$ es un término genérico de una condición patológica que se utiliza para describir situaciones que causan el crecimiento excesivo y sobredesarrollo del cóndilo mandibular, repercutiendo así también en la mandíbula, es la responsable de alrededor del $50 \%$ de las asimetrías faciales y se presenta con mayor frecuencia entre los 11 y 30 años de edad. Se presenta un caso clínico de una paciente femenina de 21 años de edad que presentaba hiperplasia condilar unilateral izquierda con compensación alveolodentaria. Se realizó condilectomía alta con abordaje endoaural para retirar $5 \mathrm{~mm}$ de la parte superior del cóndilo y osteotomía mandibular mediante abordaje intraoral circunvestibular, con disección y preservación del nervio dentario inferior, retirando $8 \mathrm{~mm}$ del aspecto inferior de la mandíbula de acuerdo a los requerimientos estéticos. El brindar un tratamiento adecuado a la hiperplasia condilar enfocado a corregir las secuelas tanto funcionales como estéticas es de gran beneficio al paciente ya que le permite mejorar su calidad de vida, el correcto diagnóstico es vital para poder planear un tratamiento exitoso.
\end{abstract}

PALABRAS CLAVE: asimetría facial, hiperplasia condilar, tratamiento quirúrgico, condilectomia.

\section{INTRODUCCIÓN}

La hiperplasia condilar $(\mathrm{HC})$ es un término genérico utilizado para describir las condiciones que causan crecimiento excesivo y sobredesarrollo del cóndilo mandibular, es responsable de deformidad dentofacial asimétrica con afectación funcional y estética y se presenta con mayor frecuencia entre los 11 y 30 años de edad, no presenta predilección por ningún sexo ni por lado de afectación (Saridin et al., 2009; Wolford et al., 2009; Wintergerst Fisch et al., 2011; Neville et al., 2014).

La HC es conocida de distintas maneras; prognatismo mandibular desviado, gnatismo mandibular lateral, osteocondroma del cóndilo mandibular y deformidad dentofacial asimétrica clase III (Posnick, 2014).
Wolford et al. (2014) propusieron una clasificación sencilla para los distintos tipos de HC, basada en las características histopatológicas, clínicas e imagenológicas. HC tipo 1, es una aberración del crecimiento del cóndilo causando elongación condilar y mandibular (prognatismo), puede ser bilateral ( $\mathrm{HC}$ tipo 1A) o unilateral (HC tipo 1B). La HC tipo 2, es una alteración condilar con alargamiento, la $\mathrm{HC}$ tipo $2 \mathrm{~A}$ tiene un vector de crecimiento que causa elongación vertical con alargamiento del cuello y cabeza condilar, y el tipo $2 B$ presenta un crecimiento tumoral exofítico en sentido horizontal del cóndilo y puede ser causada por un osteocondroma. La HC tipo 3 son otros tumores benignos y la $\mathrm{HC}$ tipo 4 son neoplasias malignas originadas en el cóndilo que causan alargamiento. Existe demasiada controversia sobre las diferencias

\footnotetext{
${ }^{1}$ Facultad de Odontología, Universidad Juárez del Estado de Durango. Durango, Dgo. México.

2 Profesor Titular de Cirugía Maxilofacial. Dirección de Investigación de la Universidad de Cuenca, Ecuador.

${ }^{3}$ Hospital Regional "Dr. Ignacio Zaragoza", ISSSTE, Ciudad de México, México

${ }^{4}$ Facultad de Odontología, Universidad de Monterrey, Monterrey Nuevo León México.

${ }^{5}$ Departamento de Estomatología del Instituto de Ciencias Biomédicas, de la Universidad Autónoma de Ciudad Juárez, México.

${ }^{6}$ Facultad de Odontología, Universidad Juárez del Estado de Durango, Durango, Dgo. México.
} 
entre hiperplasia condilar y osteocondroma, Epker se basa únicamente en la forma del cóndilo para diferenciarlas ya que la anatomía patológica no permite hacer el diagnóstico diferencial.

Existen clasificaciones previas como la de Obwegeser \& Makek (1986), que describe 2 tipos diferentes de $\mathrm{HC}$ que se correlacionan con la clasificación de Wolford. Elongación hemimandibular $(\mathrm{EH})$ en sentido horizontal, que corresponde a la HC tipo 1, y la Hiperplasia hemimandibular $(\mathrm{HH})$ con crecimiento en sentido vertical con el maxilar siguiendo a la mandíbula creando un canteamiento en la oclusión (HC tipo 2), los autores mencionados realizaron una distinción entre una hiperplasia condilar exclusiva del cóndilo ( $\mathrm{HC}$ tipo $2 \mathrm{~A}$ ) y un tumor óseo con exostosis del cóndilo (HC tipo 2B) (Wolford et al., 2014; Obwegeser \& Makek).

En la HC tipo 2 el crecimiento es vertical, se puede apreciar una cabeza y cuello condilar que son mucho más grandes en longitud y en diámetro que lo normal, el resultado es un aumento tridimensional de un lado de la mandíbula que incluye al cóndilo, al cuello del cóndilo, la rama, el cuerpo y la sínfisis, con inversión de la escotadura pregoniaca, la anomalía termina exactamente a nivel de la sínfisis en el lado afectado y en su forma más pura esta condición no produce una desviación severa de la línea media dental o del mentón hacia el lado contralateral y presenta canteamiento del maxilar mínimo pero sin producir mordida cruzada. En la HC tipo 1 aunque el cóndilo usualmente tiene una arquitectura normal existe un aumento en la longitud de la cabeza condilar, cuello y cuerpo mandibular, ello conduce a una inclinación transversal del cuerpo mandibular con desviación del mentón y de la línea media hacia el lado contralateral (Posnick; Wolford et al., 2009, 2014).

Robert Adams en 1836 fue el primero en reportar la HC, la etiología aún no está bien establecida, sin embargo, se atribuye a factores genéticos, alteraciones en la vascularidad local, traumatismos, procesos infecciosos, alteraciones endocrinas entre otros (Bharathi et al., 2014). Las características clínicas son; asimetría facial, alteraciones en la articulación temporomandibular, maloclusiones y desviación mandibular (Mehrotra et al., 2011), la asimetría asociada a dicha condición se ha clasificado en tres categorías: hiperplasia mandibular, elongación mandibular y la combinación de las dos anteriores (Xavier et al., 2014).
El tratamiento de la hiperplasia condilar es muy variado y va acorde a la edad del paciente, las características clínicas, y los requerimientos estéticos y funcionales, así como de la actividad ósea condilar. Obwegeser \& Makek recomiendan una condilectomía alta cuando la hiperplasia hemimandibular es identificada con actividad y estado progresivo de sobrecrecimiento, dentro de los tratamientos quirúrgicos de elección se encuentran la cirugía ortognática, osteotomía mandibular y condilectomia (Alyamani \& Abuzinada, 2012). Los pacientes con asimetrías faciales sin alteración de la oclusión pueden ser tratados con osteotomía del margen inferior mandibular de acuerdo a las necesidades estéticas y severidad de la asimetría (Olate \& de Moraes, 2012).

A continuación, se presenta la descripción del tratamiento de un caso clínico de hiperplasia condilar de una joven que acudió al departamento de cirugía maxilofacial de nuestro centro hospitalario.

\section{CASO CLÍNICO}

Paciente femenina de 21 años de edad la cual presentaba aumento de la longitud en sentido vertical en la mandíbula del lado izquierdo, con diagnóstico presuntivo de hiperplasia condilar tipo 2 (hiperplasia hemimandibular) En la historia clínica negaba antecedentes sistémicos y/o traumáticos relacionadas con la hiperplasia condilar, a la exploración física era evidente la asimetría facial con elongación unilateral y crecimiento vertical mandibular izquierdo, desviación de la línea media maxilomandibular y del mentón hacia la derecha (Fig. 1A,B), con evidente canteamiento maxilar y compensación alveolodentaria. A la palpación sin ruidos ni chasquidos articulares, en la exploración intraoral presentaba oclusión conservada (Fig. 1C), ligera mordida abierta del lado izquierdo, desviación de la línea media mandibular de $1 \mathrm{~mm}$ y apertura máxima bucal de $35 \mathrm{~mm}$.

En estudios radiográficos de ortopantomografía y cefalometría posteroanterior (Fig. 2) se evidenciaba asimetría facial con altura vertical aumentada de toda la mandíbula en el lado izquierdo con sobre-desarrollo vertical compensatorio del maxilar, así como aumento del diámetro de la cabeza condilar. En la tomografía computarizada por emisión individual de fotones (SPECT) había hipercaptación en la articulación del lado izquierdo, corroborando el diagnóstico de hiperplasia condilar tipo 2. 


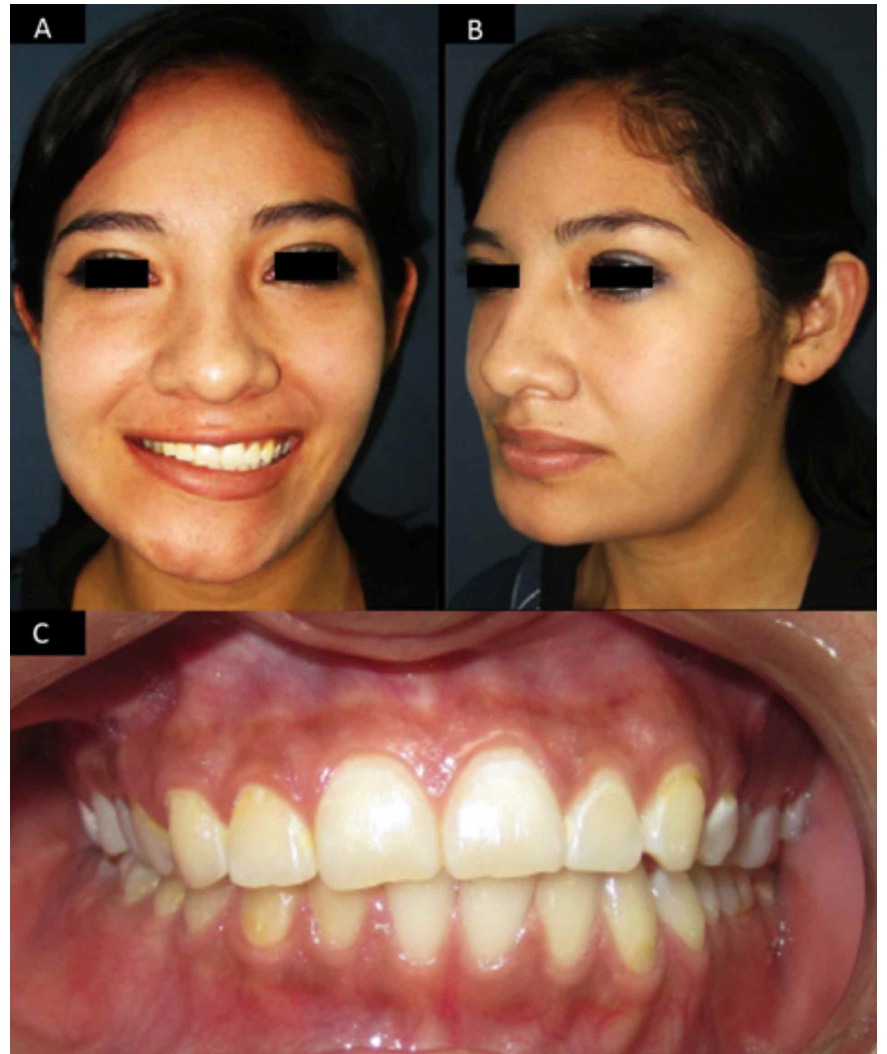

Fig. 1. A y B) Vista frontal y de tres cuartos en donde se observa una asimetría notoria en la paciente, C) Condiciones en las que se encontraba la oclusión del paciente previo al tratamiento quirúrgico.
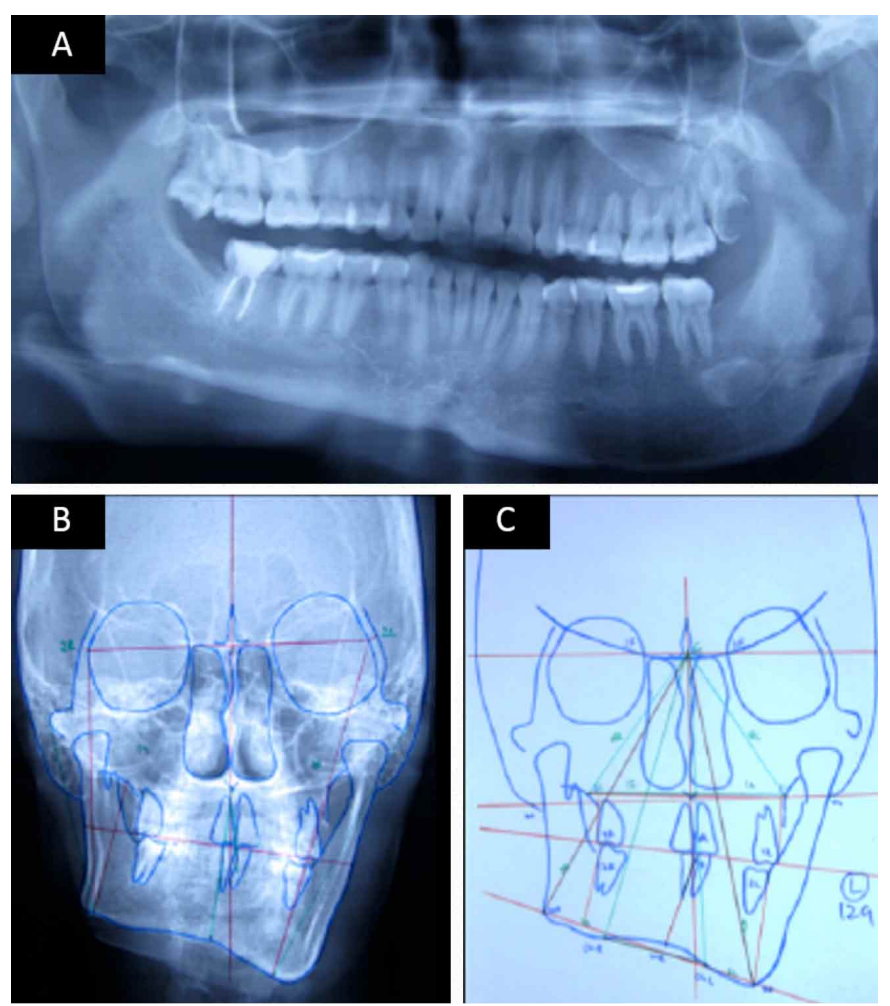

Se procedió a realizar condilectomía alta y osteotomía mandibular basal bajo anestesia general, mediante abordaje endoaural (Fig. 3) se disecó hasta la cápsula articular para abordar el espacio superior y se incidió el ligamento lateral discal para exponer totalmente el cóndilo mandibular, se colocó un separador maleable como protección en el aspecto medial y se realizó la condilectomía alta, retirando $5 \mathrm{~mm}$ del aspecto superior de la cabeza del cóndilo con una fresa quirúrgica de fisura, observando sangrado del hueso medular y con cuidado de no dañar estructuras adyacentes se reposicionó el disco y el cóndilo. Se reconstruyó el ligamento lateral discal, la capsula articular y se suturó por planos. Se procedió al abordaje intraoral mediante incisión circunvestibular mandibular izquierda disecando y respetando ramas labiales y el nervio mentoniano, se disecó la porción vestibular e inferior del cuerpo y sínfisis mandibular para realizar la osteotomía del reborde basal de $8 \mathrm{~mm}$, se suturó con sutura de ácido poliglicólico 4-0. Se realizó manipulación manual de la mandíbula para corroborar movimientos articulares adecuados con oclusión estable, se dejó en posición pasiva sin fijación intermaxilar.

La paciente se sometió a fisioterapia para rehabilitación de la articulación temporomandibular (ATM) sin necesidad de tratamiento ortodóntico. Se envió el espécimen para estudio histopatológico en donde el resultado fue hiperplasia condilar tipo 2. Se realizó la correlación clínica-imagenológica e histopatológica.

En el control posoperatorio (Fig. 4) la paciente mostraba mejoría estética con armonía facial y oclusión conservada, sin recurrencia del crecimiento hemimandibular, adecuados movimientos excéntricos mandibulares sin datos de luxación, ruidos o chasquidos articulares, la ortopantomografía de control indicaba adecuada conformación ósea con significativa mejoría en la simetría mandibular con readaptación del cóndilo a la fosa glenoidea izquierda.

Fig. 2. A) Ortopantomografía prequirúrgica con elongación vertical del cuello y cabeza condilar izquierda, con altura vertical aumentada de toda la mandíbula en el lado afectado con canteamiento vertical compensatorio del maxilar y compensaciones dentales B y C) Proyección posteroanterior con trazado cefalométrico evidenciando asimetría facial con sobredesarrollo vertical de la rama y cuerpo mandibular del lado izquierdo con plano oclusal canteado y con una relación oclusal aceptable. 

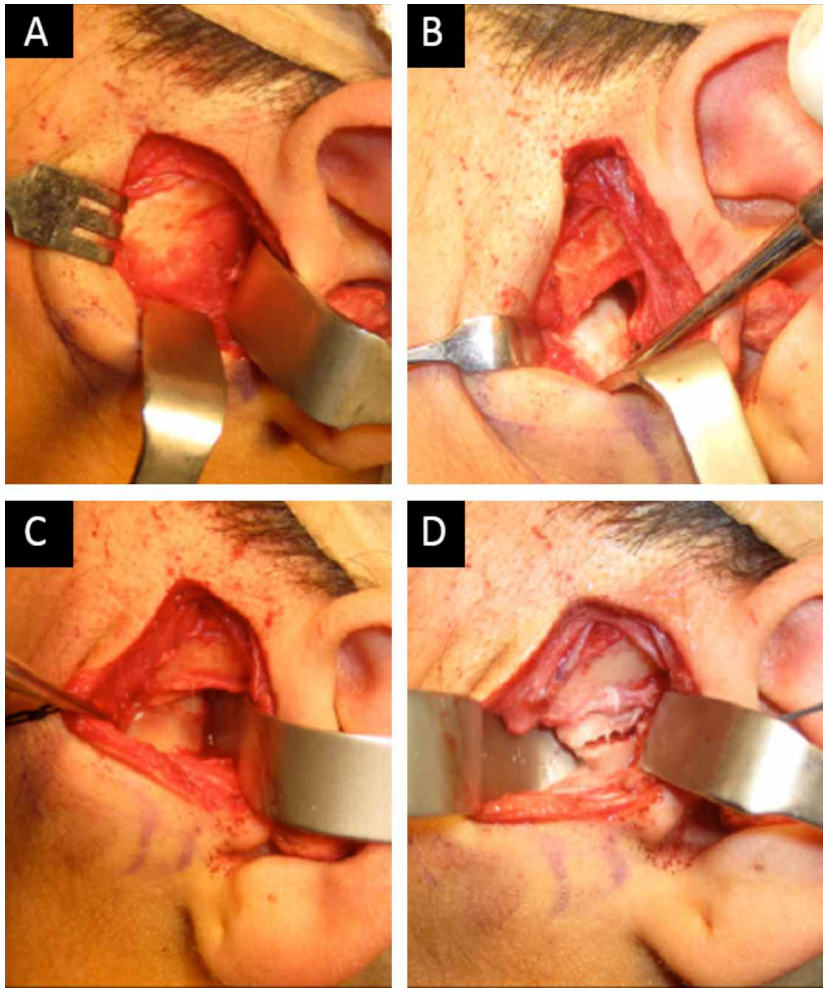

Fig. 3. Abordaje endoaural, exposición de la cápsula y rasurado condilar.
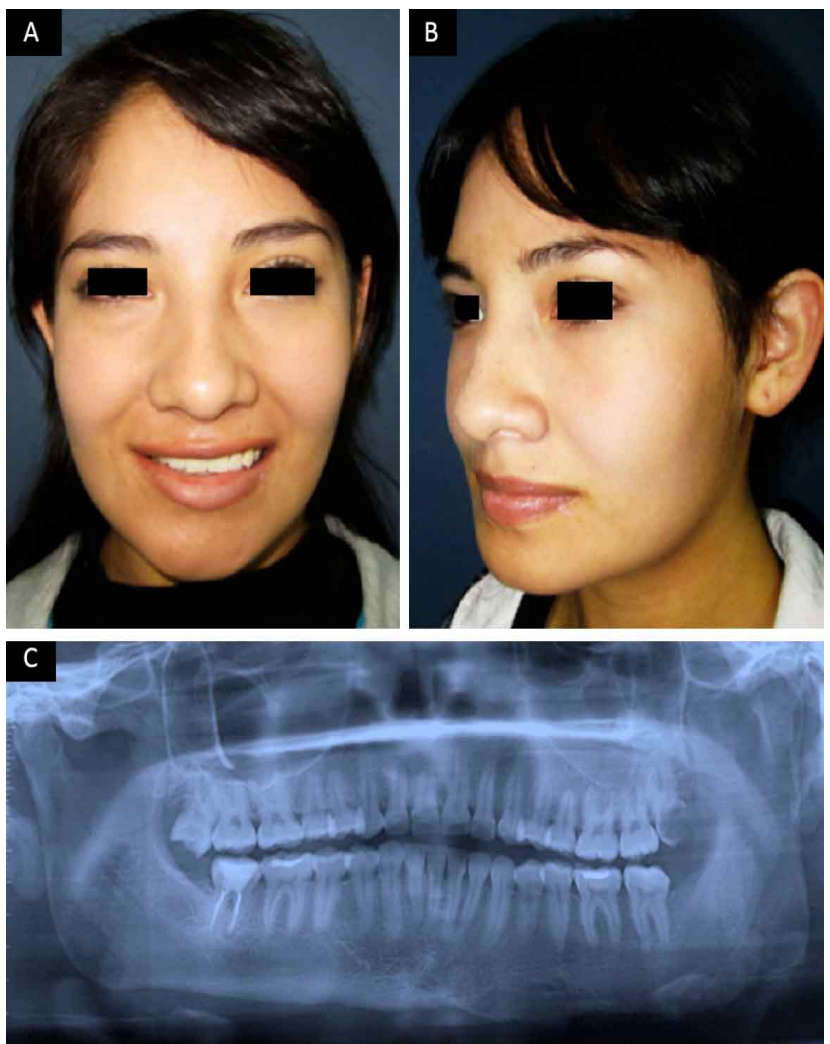

Fig. 4. A y B) Control posoperatorio, vista frontal y tres cuartos con adecuada simetría, C) Ortopantomografía de control.

\section{DISCUSIÓN}

Existe un signo diferenciador entre la $\mathrm{HC}$ tipo 1 y tipo2, en la HC tipo 2 se presenta un desplazamiento caudal del canal dentario en el lado afectado.

En la histopatología se observó una masa bien circunscrita de tejido cartilaginoso hialino, maduro con lagunas de condrocitos pequeños y un citoplasma pálido alrededor del núcleo, así como presencia de tejido óseo compacto irregular (Neville et al.), lo cual confirmo el diagnóstico de osteocondroma. Por lo cual nosotros creemos que es fundamental realizar una correlación clínica-imagenológica e histopatológica para establecer un diagnóstico certero.

Raijmakers et al. (2012) realizaron un meta-análisis para determinar la predisposición del sexo en la presencia de hiperplasia condilar unilateral, en el que reportaron una predilección del 64 \% en el sexo femenino, concluyendo que éste es un factor de riesgo para el desarrollo de esta patología. El caso clínico que presentamos es de una paciente de 21 años de edad, si bien las mujeres según algunos reportes son más afectadas consideramos que falta literatura sólida que estudie si existe una relación causal entre el sexo y la hiperplasia condilar unilateral.

Dentro de las principales características clínicas que presenta esta patología se encuentran; mayor longitud de la cabeza, cuello del cóndilo y de la rama mandibular, al igual que un alargamiento compensatorio del maxilar y cambios oclusales, información que concuerda con las alteraciones óseas observadas en los estudios imagenelógicos realizados a nuestra paciente (Gn et al., 2015).

Los principales métodos diagnósticos con los que se cuentan en la actualidad van desde radiografías convencionales como la ortopantomografía hasta el SPECT, Kubota et al. (1999) mencionaban que la radiografía panorámica era una de las herramientas más utilizadas que permite analizar la asimetría entre ambos planos de la mandíbula pudiendo comparar tamaño, altura y forma de las estructuras mandibulares; el estudio radiográfico realizado a nuestra paciente fue de gran utilidad al evidenciar una altura mandibular alterada así como cambios en el tamaño del cóndilo. Las proyecciones postero-anteriores permiten comparar las estructuras de lado derecho e izquierdo, nosotros pudimos apreciar una marcada desviación mentoniana hacia la derecha con un evidente sobre- 
desarrollo de la rama y cuerpo mandibular (López \& Corral, 2015).

Cabe mencionar que uno de los estudios claves que determinan el tratamiento de la hiperplasia condilar es el SPECT el cual permite observar la hiperactividad ósea, muestra superioridad frente a las imágenes planares gracias a su mayor sensibilidad y posibilidad de cuantificación, nosotros al valorar este estudio pudimos detectar una marcada hipercaptación de la ATM del lado izquierdo (Villanueva-Alcojol et al., 2011). Dentro de los principales diagnósticos diferenciales de la hiperplasia condilar se encuentran la microsomia hemifacial, la atrofia hemifacial, tumores óseos, entre otros, por lo que el apoyarnos de estudios imagenológicos nos permitió fijar el plan de tratamiento a seguir.

Existen diversos tratamientos para la hiperplasia condilar y estos se ven determinados por la actividad del cóndilo, Lippold et al. (2007) señalaron que la resección de este está indicada para limitar el aumento de la asimetría facial durante su fase activa de crecimiento. El Mozen et al. (2015) realizaron un estudio donde compararon pacientes que se les había realizado remodelado condilar con o sin tratamiento de ortodoncia posterior a la cirugía donde concluyeron que la condilectomia alta es un tratamiento efectivo al mejorar la oclusión funcional y la estética facial, el tratamiento de ortodoncia podría ser más efectivo al corregir el hueso alveolar y al establecer una oclusión estable.

La elección de tratamiento mediante condilectomia alta fue debido a que esta es una de las técnicas quirúrgicas que cuentan con mayor soporte en la literatura al eliminar la porción dañada del cóndilo y conservar la funcionalidad del mismo, la osteotomía basal mandibular está indicada por fines estéticos para tratar de recobrar la armonía facial

Olate et al. (2014) reportaron que aquellos pacientes a los que se les realizo osteotomía condilar se les dio seguimiento de 3 a 24 meses, todo este tiempo los pacientes reportaron ausencia de sintomatología dolorosa, pudiendo llevar a cabo sus actividades diarias, sin mostrar cambios en la asimetría ósea. En el caso que mostramos en el control posoperatorio se evidencio significativa mejoría de la armonía facial, así como ausencia total de sintomatología por lo que proponemos el uso de la osteotomía condilar con el apoyo de fisioterapia para la rehabilitación de la articulación temporomandibular.

\section{CONCLUSIONES}

El brindar un tratamiento adecuado a la hiperplasia condilar enfocado a corregir las secuelas tanto funcionales como estéticas es de gran beneficio al paciente ya que le permite mejorar su calidad de vida, el cirujano maxilofacial debe estar consciente de que el correcto diagnóstico es vital para poder planear un tratamiento exitoso.

BARAJAS-PÉREZ, V. H.; CAZAR-ALMACHE, M. E.; MIRANDA-VILLASANA, J. E.; RECENDEZ-SANTILLAN, N. J.; CUEVAS-GONZÁLEZ, J. C. \& ZAMBRANO, G. G. Surgical management of mandibular condylar hyperplasia type 2: Case report. Int. J. Odontostomat., 13(1):5-10, 2019.

ABSTRACT: Condylar hyperplasia $(\mathrm{CH})$ is a generic term for a pathological situation that is used to describe conditions that cause excessive growth and overdevelopment of the mandibular condyle and also impacting on the jaw, this bone formation is responsible about of $50 \%$ of all deformities facial and it occurs most frequently between 11-30 years old. This case report is about a 21-years old female who showed unilateral condylar hyperplasia of left side with alveolodentary compensatory. Was carried out high condilectomy through an endoaural approach removing $5 \mathrm{~mm}$ of the upper condyle and the mandibular osteotomy was performed through intraoral approach with dissection and preservation of the inferior alveolar nerve, removing $8 \mathrm{~mm}$ of the inferior aspect of the mandible according to the aesthetic requirements. An appropriate treatment for the condylar hyperplasia focused on correcting the side effects such as functional or aesthetic it's of great benefit for the patient because it allows improve their quality of life. A correct diagnosis is very important to planning a successful treatment.

KEY WORDS: facial asymmetry, condylar hyperplasia, surgical treatment, condylectomy.

\section{REFERENCIAS BIBLIOGRÁFICAS}

Alyamani, A. \& Abuzinada, S. Management of patients with condylar hyperplasia: A diverse experience with 18 patients. Ann. Maxillofac. Surg., 2(1):17-23, 2012.

Bharathi, S. C.; Senthilnathan, S.; Kumar, L. D.; Mohan, A. C. \& Taranath, M. Unilateral condylar hyperplasia: A case report and review of literature. J. Int. Soc. Prev. Community Dent., 4(1):6770, 2014.

El Mozen, L. A.; Meng, Q. G.; Li, Y. J.; Long, X. \& Chen, G. X. Condylar and occlusal changes after high condylectomy and orthodontic treatment for condylar hyperplasia. J. Huazhong Univ. Sci. Technolog. Med. Sci., 35(2):265-70, 2015. 
Gn, S.; Sharma, M. L.; Jk, D. R.; Goel, S. \& Srivastava, S. Facial asymmetry in young adults with condylar hyperplasia-unusual changes in the facial bones. J. Clin. Diagn. Res., 9(1):ZD21-3, 2015.

Kubota, Y.; Takenoshita, Y.; Takamori, K.; Kanamoto, M. \& Shirasuna, K. Levandoski panographic analysis in the diagnosis of hyperplasia of the coronoid process. Br. J. Oral Maxillofac. Surg., 37(5):409-11, 1999

Lippold, C.; Kruse-Losler, B.; Danesh, G.; Joos, U. \& Meyer, U. Treatment of hemimandibular hyperplasia: the biological basis of condylectomy. Br. J. Oral Maxillofac. Surg., 45(5):353-60, 2007.

López B., D. F. \& Corral S., C. M. Hiperplasia condilar: características, manifestaciones, diagnóstico y tratamiento. Revisión de tema. Rev. Fac. Odontol. Univ. Antioq., 26(2):425-46, 2015.

Mehrotra, D.; Dhasmana, S.; Kamboj, M. \& Gambhir, G. Condylar hyperplasia and facial asymmetry: report of five cases. J. Maxillofac. Oral Surg., 10(1):50-6, 2011.

Neville, B.; Damm, D.; Allen, C. \& Bouquot, J. Oral and Maxillofacial Pathology. $3^{\text {rd }}$ ed. Philadelphia, Saunders, 2014. pp.656-7.

Obwegeser, H. L. \& Makek, M. S. Hemimandibular hyperplasia-hemimandibular elongation. J. Maxillofac. Surg., 14(4):183-208, 1986.

Olate, S. \& de Moraes, M. Asymmetric facial deformity. Role of condylar hyperplasia. Int. J. Odontostomat., 6(3):337-47, 2012.

Olate, S.; Martinez, F.; Uribe, F.; Pozzer, L.; Cavalieri-Pereira, L. \& de Moraes, M. TMJ function after partial condylectomy in active mandibular condylar hyperplasia. Int. J. Clin. Exp. Med., 7(3):7759, 2014.

Posnick, J. C. Asymmetric Mandibular Excess Growth Patterns. En: Duncan, L. (Ed.). Orthognathic Surgery: Principles and Practice. Missouri, Elsevier, 2014. pp.807-65.

Raijmakers, P. G.; Karssemakers, L. H. \& Tuinzing, D. B. Female predominance and effect of gender on unilateral condylar hyperplasia: a review and meta-analysis. J. Oral Maxillofac. Surg., 70(1):e72-6, 2012.

Saridin, C. P.; Raijmakers, P. G.; Al Shamma, S.; Tuinzing, D. B. \& Becking, A. G. Comparison of different analytical methods used for analyzing SPECT scans of patients with unilateral condylar hyperactivity. Int. J. Oral Maxillofac. Surg., 38(9):942-6, 2009.

Villanueva-Alcojol, L.; Monje, F. \& González-García, R. Hyperplasia of the mandibular condyle: clinical, histopathologic, and treatment considerations in a series of 36 patients. J. Oral Maxillofac. Surg., 69(2):447-55, 2011.

Wintergerst Fisch, A.; Iturralde Espinosa, C. \& Reinoso Quezada, $\mathrm{S}$. Asimetría facial secundaria a hiperplasia condilar mandibular. Reporte de un caso. Rev. Odontol. Mex., 15(4):251-6, 2011.

Wolford, L. M.; Morales-Ryan, C. A.; García-Morales, P. \& Pérez, D. Surgical management of mandibular condylar hyperplasia type 1. Proc. (Bayl Univ. Med. Cent.), 22(4):321-9, 2009.

Wolford, L. M.; Movahed, R. \& Pérez, D. E. A classification system for conditions causing condylar hyperplasia. J. Oral Maxillofac. Surg., 72(3):567-95, 2014.

Xavier, S. P.; Santos, T. de S.; Silva, E. R.; Faria, A. C. \& de Mello Filho, F. V. Two-stage treatment of facial asymmetry caused by unilateral condylar hyperplasia. Braz. Dent. J., 25(3):257-60, 2014.
Dirección para Correspondencia

Dr. Juan Carlos Cuevas González

Departamento de Estomatología

Universidad Autónoma de Ciudad Juárez

Anillo Envolvente del Pronaf s/n

Zona Pronaf, 32315

Cd Juárez

Chihuahua

MÉXICO

Email: cuevas_gonzalez@hotmail.com

Recibido : 02-06-2018

Aceptado : 27-09-2018 